\title{
Importance of Epigenetic in Plants
}

\author{
Madhusudhan L* \\ Department of Biotechnology, Joginpally B R Engineering College, JNTU, Telangana, India
}

Epigenetic is one of the most important topics in the field of plant genetics. It is a promising aspect to impart plant stress in different plant species. Many scientific studies have been supporting the development of plant genetics. Epigenetic is an important aspect to solve the problems in transgenic plants, with suitable expression from new transgenic segments. In this paper some of the important aspects of epigenetic are enlighten to support the future research studies. Plants gained the ability to change their response to environmental stimuli. Epigenetic changes in gene expression have fascinated scientists over several decades. These processes received particular attention in plants, where they can result in beautiful variations in conspicuous phenotypes such as pigmentation. Epigenetic control is also a key issue in the development of transgenic plants with appropriate expression from newly introduced transgene segments. The term 'epigenetic' refers to heritable gene expression patterns determined by how the DNA of a gene is packaged rather than its primary DNA sequence. Genes are tightly packed within DNA and they are not available to the transcription machinery and are expressed very poor. Normally the patterns of DNA packaging are carefully controlled to give predictable patterns of gene expression. However, the process can occasionally go awry to cause altered gene expression. This primer will focus on well characterized examples of epigenetic changes in plants that shed light on the mechanisms underlying this fundamental gene control process. [1]. Defense, plants evolved sophisticated mechanisms to respond and acclimatize to these stresses by prompt and harmonized changes at transcriptional and post-transcriptional levels of whole gene complexes [2]. According to Richards, the range of epigenetic variation in relation to genotypic context could be categorizedas three: obligatory, facilitated and pure. Whereas the obligate epigenetic variation is completely dependent on genetic variation, facilitated epigenetic variation is semiindependent of genetic variation, and pure epigenetic variation is completely independent of genetic variation [3].

\section{Role of epigenetics and plant genetics}

The effect of epigenetic over the plants and crops such as maizehas many strains with striking patterns of kernel or plant pigmentation was selected for cultivation. These strains were provided a rich source of epigenetic variation in pigment gene expression. One such case that has been examined at the molecular level is the expression of a transcription factor gene that controls pigment synthesis [1]. According to Judith Bender the variations in epigenetic results to pigment gene expression. Cotton is not only the most important source of renewal textile fibers, but also an important model for studying cell fate determination and polyploidy effects on gene expression and evolution of domestication traits. The combination of $\mathrm{A}$ and $\mathrm{D}$-progenitor genomes into allotetraploid cotton induces intergenomic interactions and epigenetic effects, leading to the unequal expression of homologous genes. Small RNAs regulate the expression of transcription and signaling factors related to cellular growth, development and adaptation.

Epigenetic changes in DNA and chromatin affect the genes and transposons activity. Epigenetic controls the time of flowering, parent of origin imprinting, paramutation and transposon silencing. Genome studies of epigenetic marks revealed the critical role of small interfering RNAs in maintaining epigenetic states [4].
Tomato is an important vegetable all over the world and it is important to carry research regarding it, to develop its growth. A novel insight into gene regulatory mechanism was presented by an epigenetic modification study of a tomatoprotein-coding non-transposon ASR1 (Abscisic acid stress, ripening 1) epiallele, demonstrating its role in DNA methylation during water deficit stress [2]. According to Gonzalez et al. DNA methylation was occurred with possible changes in epigenetics of tomato.

From the past few years there wasan increase in the modification of agriculture crops with transgenes which express desirable traits. However, a frequent stumbling block is the unwanted silencing of the transgene. Many studies of silenced transgenes and silenced endogenous sequences showed that repeated sequence arrays, in particular inverted repeats are most likely silencing. Thus, selection of transgenic plants with singlecopy transgene insertions is the first line of defense against silencing [1]. An interesting approach to identify epigenetically controlled probable gene sets in response to drought stress was performed by Shaik and Ramakrishna [5]. Epigenetics and plant genetics are the most important areas to develop agriculture and the plant development.

\section{Conclusion}

From the past few years topic of epigenetic became very popular in research studies of plant genetics. The most important aspect of the epigenetic is the mechanism; these mechanisms play an important role in gene expression by the enzyme actions which exhibit the positive result. This study states that the epigenetics supports the plants with metabolic variations and activates the gene regulation.

\section{References}

1. Judith bender (2002) Plant epigenetics.

2. Golldack D, Luking I, Yang O (2011) Plant tolerance to drought and salinity: stress regulating transcription factors and their functional significance in the cellular transcriptional network. Plant Cell Rep 30: 1383-1391.

3. Richards EJ (2006) Inherited epigenetic variation-revisiting soft inheritance. Nat Rev Genet 7: 395-401.

4. Williams SP (2013) Epigenetics. Pnas 110: 3209.

5. Shaik R, Ramakrishna W (2012) Bioinformatic analysis of epigenetic and micro-RNA mediated regulation of drought responsive genes in rice. PLoS ONE 7: e49331.

*Corresponding author: Madhusudhan L, Department of Biotechnology, Joginpally B R Engineering College, JNTU, Telangana, India, E-mail: madhu.sl@rediffmail.com

Received: April 15, 2015; Accepted: April 27, 2015; Published: May 04, 2015

Citation: Madhusudhan $L$ (2015) Importance of Epigenetic in Plants. J Bioengineer \& Biomedical Sci 5: 151. doi:10.4172/2155-9538.1000151

Copyright: @ 2015 Madhusudhan L. This is an open-access article distributed under the terms of the Creative Commons Attribution License, which permits unrestricted use, distribution, and reproduction in any medium, provided the original author and source are credited. 